\title{
Norois
}

Environnement, aménagement, société

$236 \mid 2015$

Présence chinoise en Arctique, nautisme, ville-port, vulnérabilité, inondation

\section{La stratégie de la Chine en Arctique : agressive ou opportuniste?}

China's strategy in the Arctic: aggressive or opportunist?

Frédéric Lasserre, Olga V. Alexeeva et Linyan Huang

\section{(2) OpenEdition}

\section{Journals}

Édition électronique

URL : https://journals.openedition.org/norois/5681

DOI : $10.4000 /$ norois. 5681

ISBN : 978-2-7535-4945-6

ISSN : 1760-8546

Éditeur

Presses universitaires de Rennes

Édition imprimée

Date de publication : 30 décembre 2015

Pagination : 07-24

ISBN : 978-2-7535-4903-6

ISSN : 0029-182X

Référence électronique

Frédéric Lasserre, Olga V. Alexeeva et Linyan Huang, « La stratégie de la Chine en Arctique : agressive ou opportuniste? », Norois [En ligne], 236 | 2015, mis en ligne le 30 décembre 2017, consulté le 01 février 2022. URL : http://journals.openedition.org/norois/5681 ; DOI : https://doi.org/10.4000/norois 5681

(c) Tous droits réservés 


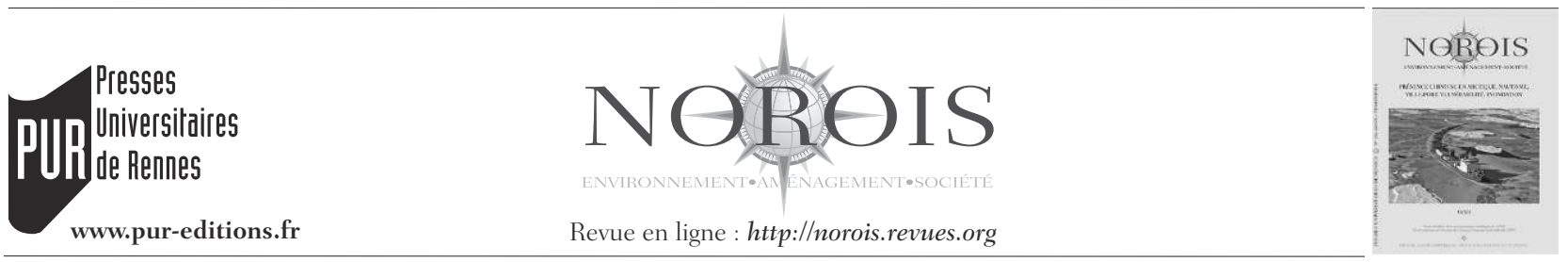

\title{
La stratégie de la Chine en Arctique : agressive ou opportuniste?
}

\author{
China's Strategy in the Arctic: Aggressive or Opportunist?
}

Frédéric Lasserre*a ${ }^{* a}$ Olga V. AleXeeva ${ }^{\mathrm{b}}$, Linyan Huang ${ }^{\mathrm{c}}$

\footnotetext{
* Auteur correspondant

a Directeur de projet, ArcticNet. Professeur au Département de géographie, Université Laval, Québec. (Frederic.

Lasserre@ggr.ulaval.ca)

b Professeur d'histoire de la Chine, Département d'histoire, Université de Québec à Montréal (UQÀM).

c Candidate au doctorat, Université Laval, Québec.
}

Résumé : Dans un contexte de bouleversements climatiques dans l'Arctique, la fonte rapide du pergélisol, le recul des glaciers et la fonte de la banquise créent des occasions stratégiques et économiques qui n’ont pas échappé aux États riverains. L'intérêt pour la région ne se limite pas aux pays directement concernés. La Chine, par exemple, sans accès direct à l'Arctique, a adopté une attitude officielle très prudente; cependant, ses efforts diplomatiques, économiques, politiques et scientifiques dans cette région suscitent des réactions négatives de la part des médias occidentaux. Ils dressent souvent un portrait d'une Chine ambitieuse et arrogante, prête à bousculer la souveraineté des pays arctiques pour défendre ses intérêts dans cette région. Dans cette optique, il paraît pertinent d'analyser les activités de la Chine en Arctique et de tenter d'identifier quelle est la stratégie de Pékin dans cette zone polaire.

Abstract: In a context of climatic upheavals in the Arctic, the fast melting of permafrost, the decline of glaciers and the melting of sea ice created strategic and economic opportunities which did not escape the riparian States. The interest for the region goes beyond its shores. China, for example, without direct access to the Arctic, adopted a very careful official attitude; however, its diplomatic, economic, political and scientific efforts in this region arouse negative reactions among western media. They often draw up a portrait of an ambitious and arrogant China, ready to push aside the sovereignty of the Arctic countries to defend its interests in the Arctic. From this perspective, it seems relevant to analyze China's activities in the region and try to assess Beijing's strategy in the Arctic.

Mots clés : Chine - Arctique - diplomatie - ressources - navigation - routes maritimes

Keywords: China - Arctic - diplomacy - resources - shipping - maritime routes

\section{INTRODUCTION}

Le premier transit du passage du Nord-Est, au nord des côtes sibériennes en août 2013, par un navire de commerce chinois, le Yongsheng de la compagnie d'État Cosco, illustre-t-il les ambitions arctiques de Pékin? Dans le contexte des change- ments climatiques, on observe un intérêt grandissant pour l'Arctique dans les discours des médias sur les enjeux de souveraineté, sur l'abondance supposée de ressources naturelles et sur l'ouverture possible de nouvelles routes maritimes (Holmes, 2008; Grupta, 2009, p. 174-177; Lasserre, 2010). La 
région de l'Arctique concerne huit pays, dont cinq, le Canada, la Russie, les États-Unis, la Norvège et le Danemark (via le Groenland) ${ }^{1}$, sont directement riverains de l'océan du même nom. La fonte de la banquise suscite d'importants débats et de scénarios sur les occasions économiques et stratégiques dans les pays avoisinants en quête, d'une part, de ressources naturelles et, d'autre part, de profits commerciaux que pourrait induire la mise en service d'une route maritime. L'Arctique recèlerait environ $30 \%$ du gaz naturel et $13 \%$ du pétrole à découvrir (USGS, 2008), mais l'enthousiasme des compagnies pétrolières s'est érodé au cours des derniers mois de 2014 avec la chute des cours mondiaux et, surtout, la prise de conscience des grandes difficultés techniques que représente l'exploitation dans des conditions qui demeurent très difficiles. Les routes maritimes arctiques, plus courtes mais pas nécessairement plus rapides, pourraient quant à elles offrir des gains significatifs par rapport aux itinéraires alternatifs via Suez ou Panama (Li, 2009; Lasserre, 2010b; Peresipkin et Iakovlev, 2006).

Toutefois, l'intérêt pour la région arctique ne se limite pas aux pays de la région. D'autres pays y voient également un enjeu géostratégique et sont parfois décrits comme nourrissant des convoitises à l'endroit de l'Arctique. Nombre d'États non-arctiques ont ainsi été admis comme observateurs permanents au sein du Conseil de l'Arctique, organisme de coopération politique fondé en 1996, parmi lesquels notamment l'Italie, la France, le RoyaumeUni, le Japon, la Corée du Sud, Singapour, l'Inde et la Chine. Le Japon, qui n'est pas un État riverain de l'Arctique, a déclaré dès 2009 se sentir concerné par les problèmes et les mutations de cette région du monde, surtout en ce qui concerne le développement du transport maritime et des activités de pêche (Weese, 2010). Singapour a également manifesté un intérêt pour le transport maritime dans la région lors du sommet de l'Arctic Circle en novembre 2014 à Reykjavik.

La Chine, qui n'a pas non plus d'accès géographique direct sur l'Arctique, a adopté une attitude diplomatique très prudente par rapport à cette région du monde (Ministry of Foreign Affairs of the PRC,

1. L'Islande, au nord de l'Atlantique nord, n'est pas considérée par les pays du Conseil de l'Arctique comme étant riveraine de l'océan Arctique, ce que conteste Reykjavik; une bonne part de ses zones économiques exclusives (ZEE) se trouvent au nord du cercle arctique.
2010). Toutefois, sa présence en Arctique semble de plus en plus affirmée, et de vifs mouvements d'opinion traversent tant la communauté universitaire chinoise que son opinion publique, relayée par les médias, quant à la place légitime qui devrait incomber à la Chine en Arctique. Nombre de chercheurs chinois décrivent ainsi la Chine comme un État du «proche-Arctique » [« near Arctic »] (Rainwater, 2012; IISS, 2014), comme pour justifier l'intérêt croissant qu'ils estiment légitime pour la Chine dans cette région.

En quelques années, Pékin a réussi à mettre en place et à conduire avec succès un vaste programme de recherches scientifiques indépendantes ${ }^{2}$ tout en ayant très peu d'expérience préalable de recherches en cette zone géographique. En parallèle, la Chine a fait un effort considérable pour tisser des liens politiques et économiques avec les petits pays arctiques, mais aussi pour inclure la problématique arctique dans l'agenda de discussions diplomatiques avec la Russie et le Canada (Ministry of Foreign Affairs of the PRC, 2011). Des entreprises minières chinoises sont actives au Groenland et au Canada, et les entreprises pétrolières chinoises courtisent leurs homologues russes.

Ces efforts chinois, depuis 2009 environ, suscitent des réactions négatives de la part des médias ou de certains analystes occidentaux, qui dressent souvent un portrait d'une Chine ambitieuse et arrogante prête à bousculer l'ordre juridique établi pour défendre ses intérêts en Arctique et qui n’hésiterait pas à bousculer la souveraineté des pays de la région (voir par exemple Edmonton Journal 2007; New York Times 2012; pour une analyse, voir Wright DC, 2011, 2011b, 2013; Wright TC, 2013; Beck, 2014; Peng et Wegge, 2014), mettant en exergue des articles scientifiques qui ne traduisent pas nécessairement la position officielle du gouvernement chinois, ou mettant en exergue une déclaration, par exemple « Pékin veut une part des ressources arctiques, par n'importe quel moyen » (cité par Peng et Wegge, 2014, sans que l'on sache s'il s'agit d'une position isolée ou non).

Dans cette optique, il paraît pertinent de dresser un bilan de la présence chinoise en Arctique et de

\footnotetext{
2. Les résultats de ces recherches dans les différents domaines de la science (climatologie, géologie, biologie, etc.) sont publiés non seulement en Chine mais aussi dans les revues internationales d'une grande renommée scientifique, voir, par exemple, WEI Juncai et al., 2010.
} 
se pencher sur l'analyse de sa politique envers cette région du monde, dont les ressources naturelles et les routes maritimes potentielles suscitent beaucoup de spéculations sur les convoitises des puissances régionales et mondiales, soucieuses de leur sécurité énergétique et de l'avenir de leur développement économique qui en découle. L'objectif de cet article est de donner un aperçu des activités de la Chine en Arctique, et de tenter d'identifier quelle est la stratégie de Pékin dans cette zone polaire.

\section{UN INTÉRÊT SCIENTIFIQUE ANCIEN de la Chine pour les Régions POLAIRES}

L'intérêt de la Chine pour l'Arctique semble être un phénomène assez récent. L'une des premières publications de recherches qui a attiré l'attention de la communauté internationale envers la présence croissante de la Chine fut le rapport de l'Institut international de recherche pour la paix de Stockholm (SIPRI). Intitulé China prepares for an ice-free Arctic, ce rapport analyse les activités chinoises en Arctique ainsi que l'évolution du discours officiel de Pékin concernant les enjeux énergétiques et commerciaux de cette région (Jakobson, 2010). Depuis, la Chine a fait objet d'un certain nombre d'articles de presse et de reportages, ainsi que de publications académiques analysant les aspirations de Pékin à devenir l'un des principaux acteurs en Arctique et à participer de manière active à la gestion des ressources et au débat sur la gouvernance de cette zone géographique (Alexeeva et Lasserre, 2015).

\section{Quelle production scientifique chinoise au sujet de l'Arctique?}

L'étude détaillée des activités de la Chine en Arctique montre que l'intérêt de Pékin envers cette région n'est pas aussi récent et remonte aux années 1980. Le programme officiel chinois de recherches arctiques a formellement débuté en 1989 avec la fondation de l'Institut Chinois de recherches polaires à Shanghai (Centre de recherches polaires de Chine, 2007). Selon la plus grande base de données chinoise - Wanfang Data ${ }^{3}$ [万方数据 - wan-

\footnotetext{
3. Wanfang data est la première base de données qui a été créée en Chine dans les années 1950. Au début, elle avait pour but de numériser les informations concernant des entreprises et leurs produits et a été fondé
}

fang shuju] - les premiers travaux de recherche sur l'Arctique ont débuté à la fin des années 1980. En 1988, l'Académie des Sciences chinoise a aussi commencé à publier une nouvelle revue trimestrielle entièrement consacrée aux problématiques liées à l'Arctique et l'Antarctique, Beiji yanjiu [极地 研究] ou Chinese Journal of Polar Research.

Depuis la fin des années 1980, les différentes revues académiques chinoises ont publié plusieurs centaines d'articles de chercheurs chinois, toutes disciplines confondues, consacrés à l'Arctique. La plupart d'entre eux traitent essentiellement de sujets relevant des sciences exactes - les problèmes liés au réchauffement climatique en Arctique, l'impact de ces changements sur les variations de température et le volume de précipitations en Chine etc. (par exemple Yan, 2005). Ainsi, en faisant une recherche dans la Wanfang Data, nous avons identifié 2126 entrées différentes qui comportaient le mot «Arctique » (北极 - Beiji) dans leur titre dont 1835 sont des articles publiés par une vingtaine de revues scientifiques chinoises entre 1988 et 2014. Les autres entrées sont des travaux universitaires les maîtrises et doctorats soutenus au cours de la même période (182) et des actes de colloques (109). La majorité de ces publications (30\% du total) est consacrée à toutes sortes de problèmes climatologiques et géophysiques (ex : Gong et Wang, 2003; Wu et al., 2008), les autres analysent des questions de biodiversité (15\%), d'industrie et de ressources énergétiques $(8 \%)$, de politique et de droit $(7 \%)$, d'environnement $(7 \%)$, de transport $(5 \%)$, d'économie $(4 \%)$, de santé $(1 \%)$. Finalement, un nombre important d'études est consacré aux thématiques liées à l'histoire, culture, art et langues des différentes régions et pays arctiques $(9 \%)$.

Depuis 2007, on voit apparaître des publications davantage consacrées aux différentes questions propres au domaine des sciences humaines - questions de souveraineté en Arctique, analyse de la politique arctique des pays circumpolaires, rôle de l'Arctique dans le futur développement économique et géostratégique de la Chine, etc. (Lu, 2010; Shi, 2010). On relève ainsi 157 textes (135 articles, 22 mémoires de maîtrise et de thèses et 2 actes

\footnotetext{
par l'Institut des sciences technologique et informatique en Chine. Transformée par la suite en une vaste base d'informations électronique multidisciplinaire, elle propose plusieurs collections (périodiques, thèses, archives, etc.), en ligne: [www.wanfangdata.com.cn/], c. le 7 février 2015.
} 
de colloque publiés entre 2006 et 2014, l'essentiel ayant été publié entre 2010 et 2014 , et portant sur la place de l'Arctique dans les relations internationales (46\%), sur des enjeux géopolitiques ou économiques $(8 \%)$ et sur les aspects légaux de ce dossier (46\%) dont la majorité porte sur les question de droit international (83\%). À notre connaissance, ces questions apparaissent pour la première fois en 2006 avec un article (Yu, 2006) portant sur la stratégie arctique du Canada. En 2007, Wang se penche sur les rivalités politiques et les enjeux de souveraineté en Arctique. En 2008, Liu analyse la stratégie russe en Arctique tandis que Ren et Li évoquent à nouveau les questions de souveraineté. À partir de 2009 paraissent de nombreux articles qui abordent des enjeux politiques en Arctique ou qui soulignent les intérêts de la Chine dans la région.

Les intérêts de la Chine en Arctique sont ainsi au cœur de discussions académiques où ce sujet est traité avec beaucoup moins de réserve et de prudence (Li, 2009 et 2009b; Zhang et Li, 2010; Liu et Dong, 2010; Liu et al., 2010; Cheng, 2011 ; Lu, 2011). Certains scientifiques chinois invitent leur gouvernement à changer sa position de neutralité en s'engageant davantage dans le processus de délimitation des zones de souveraineté en Arctique et du partage des ressources de cette région du monde, voire mettent en avant l'idée que les ressources arctiques doivent relever du patrimoine de l'humanité (Li, 2009; Jia, 2010 ; Dutton, 2012; Chen, 2012), positions dont la presse se fait aussi l'écho (Chang, 2010 ; Zhu, 2011 ; voir l'analyse de Wright, 2011 b), sans préciser sur quelle base juridique cette politique pourrait être menée. Jia Yu (2010), une chercheuse du département du développement de la stratégie maritime au sein de la State Oceanic Administration (SOA) ${ }^{4}$, ou Cheng Baozhi (2011), du Shanghai Institute of International Studies (SIIS), soutiennent ainsi que l'extension des plateaux continentaux au-delà des limites des zones économiques exclusives devrait être limitée, et que l'espace maritime au-delà de ces limites devrait relever du patrimoine mondial : à travers ces publications semi-officielles, c'est la notion de plateau continental étendu

4. La State Oceanic Administration [国家海洋局 Guojia Haiyang ju] est une institution officielle créée en 1964 sous tutelle du Ministère des Territoires et des Ressources pour superviser la gestion des espaces maritimes chinois, protéger les droits maritimes nationaux et organiser les activités de recherches marines en Chine. Cette organisation contrôle et coordonne également les recherches chinoises en Arctique et en Antarctique. que Pékin semblait contester sotto vocce dans son application dans l'Arctique.

Ces opinions, parfois très différentes de celles du Pékin officiel, sont publiées non seulement par les revues académiques classiques, mais aussi par les périodiques officiels chinois qui ne publient jamais de matériaux ou d'opinions non-autorisés au préalable. L'existence de ce genre de publications, qui s’inscrit dans la tendance générale de la montée du nationalisme en Chine, est délicate à interpréter : elle pourrait être un signe de la volonté de Pékin de former l'opinion publique chinoise sur l'importance des enjeux arctiques pour l'avenir socio-économique du pays et sur la nécessité pour la Chine de devenir un acteur plus actif dans cette partie du monde, ou bien tout simplement de laisser un champ d'expression à ce nationalisme, afin de détourner l'attention de l'opinion publique, sans pour autant que le gouvernement ait l'intention d'intervenir. Par ailleurs, il faut aussi se garder de croire que tous les articles scientifiques chinois font la promotion active des intérêts de la Chine dans l'Arctique : ainsi, Liu et Yang (2010) ou Mei et Wang (2010) demeurentils très modérés dans leurs propos. Enfin, il semble qu'il soit difficile d'interpréter la position du gouvernement chinois comme la remise en cause des principes du droit international : outre la reconnaissance officielle des droits souverains des États riverains en 2013 lors de son admission comme observateur permanents au sein du Conseil de l'Arctique, la Chine ne semble pas développer de raisonnement révisionniste en Arctique lorsqu'on analyse les prises de positions et les déclarations officielles (Gayazova, 2013).

\section{Le déploiement d'outils de recherche en Arctique}

L'intérêt de la Chine pour l'Arctique ne s'est pas manifesté uniquement sur les pages des articles académiques, mais aussi sur le terrain. En 1992, donc avant que l'on ne parle abondamment de l'ouverture possible des routes arctiques, Pékin a organisé son premier programme de recherches scientifiques de cinq ans dans l'océan Arctique en partenariat avec les universités allemandes de Kiel et de Brême. Ce projet a été suivi par l'admission de la Chine aux différents organismes internationaux ayant pour mission de coopération en recherche arctique, tels le 
Comité International des Sciences arctiques (CISA) ou le Pacific Arctic Group (PAG) (Xu, 2012).

L'achat d'un brise-glace (Classe polaire $5^{5}$ ) en Ukraine en 1994, baptisé Xuelong [雪龙] ou Dragon des neiges, a permis aux Chinois de monter un programme de recherches polaires indépendant et de réaliser plusieurs expéditions scientifiques en Arctique et en Antarctique. Coordonnés par l'agence nationale - Chinese Arctic and Antarctic Administration $(\mathrm{CAA})^{6}$, ces travaux de recherches ont pris une envergure impressionnante. Un deuxième brise-glace était en cours de construction fin 2014. Ainsi, outre 28 expéditions dans l'Antarctique, la Chine a préparé et conduit six expéditions en Arctique (1999, 2003, 2008, 2010, 2012 et 2014) et a fondé sa première station, «Fleuve Jaune »[黃河 - Huanghe], à Ny-Alesund, archipel du Svalbard (Norvège) (2004), qui vient compléter un réseau de stations polaires qui comprend aussi trois stations en Antarctique (Grande Muraille, fondée en 1985; Zhongshan, fondée en 1989; Kunlun, ouverte en 2009). En Chine, c'est la recherche en Antarctique, et non en Arctique, qui perçoit la part du lion des budgets de recherche polaire (près de $80 \%$, Brady 2012), ne serait-ce que parce qu'en vertu du traité de l'Antarctique (1959), Pékin n’a besoin d'aucune autorisation pour y développer bases et programmes de recherche (Keyuan, 1993; Brady, 2010). Il serait cependant inexact de penser que dès le lancement en 1981 des programmes polaires, les agences de recherche chinoises considéraient l'Antarctique comme une étape vers l'Arctique : rien ne l'atteste pour l'instant dans la littérature. Le programme de recherches chinois en Arctique est en grande partie

\footnotetext{
5. La nouvelle nomenclature internationale pour les coques de glace repose sur un classement en 7 classes, définies par l'International Association of Classification Societies (IACS). La classe polaire 7 (CP7) représentant la moins forte (équivalent approximativement à la classe 1A dans les systèmes de classification Lloyds ou Baltique : «navigation en été ou en automne dans de la glace mince de première année avec possible inclusion de glace pluriannuelle »), la plus forte étant la classe 1, des navires extrêmement puissants, capables de naviguer en toutes saisons dans la banquise. On considère que les deux premières clases (CP 7 et 6) sont des navires dits à coque renforcée, les classes 5 et au-delà étant des briseglace. IACS, Requirements concerning Polar Class, 2011, [www.iacs.org. uk/document/public/publications/unified_requirements/pdf/ur_i_pdf410. $p d f$. À titre de comparaison, le brise-glace allemand Polarstern est de classe ARC3/CP2.

6. Fondée en 1981 (cet organisme s'appelait alors le Bureau du Comité d'expédition nationale en Antarctique), Guoji haiyangju judi kaocha bangongshi [国家海洋局极地考察办公室] a cinq sections qui gèrent toutes les activités en relation avec l'Arctique et l'Antarctique, ainsi que deux bureaux à l'extérieur de la Chine - au Chili et en Australie et le centre d'entraînement à Yabuli, province de Heilongjiang, cf. le site Internet de l'organisation, Chinese Arctic and Antarctic Administration, sur [www. chinare.gov.cn/en/index.html].
}

consacré à l'étude des interactions entre l'Océan glacial arctique, les glaces maritimes et l'atmosphère, afin d'en apprendre davantage sur l'influence des changements climatiques anormaux du pôle Nord sur le climat chinois (Wang, 1988; Chen, 2003). Les voyages du brise-glace de recherche Xuelong, presque tous dans le secteur eurasiatique de l'Arctique, plus rarement en mer des Tchouktches ou de Beaufort, jamais dans ou autour de l'archipel arctique canadien ou du Groenland, semblent attester de cet intérêt plus marqué pour des recherches océanographiques arctiques en lien avec les mécanismes climatiques affectant l'Asie du Nord-Est.

En 2012, le gouvernement chinois a annoncé la construction d'un second brise-glace qui devrait permettre aux scientifiques chinois d'élargir leurs recherches polaires. Le nouveau navire devrait entrer en service en 2016 (China Daily, 2014). Il disposera de nombre d'équipements spécialisés qui aideront les chercheurs à étudier l'environnement océanique, ainsi qu'à intégrer beaucoup plus rapidement les données recueillies lors des expéditions polaires (People's Daily, 2011). De plus, en juin 2013, le gouvernement chinois a annoncé la création d'un centre de recherche arctique en partenariat avec les pays scandinaves, le China-Nordic Arctic Research Center (Barents Observer, 2013).

Ces outils de recherche traduisent certes un intérêt scientifique réel, mais présentent également l'avantage pour Pékin de fournir des outils pour alimenter une présence sur le terrain, structurant ainsi une véritable diplomatie de la recherche, comme en Antarctique où la Chine, par ailleurs, est beaucoup plus active qu'en Arctique (Alexeeva et Lasserre, 2012a, 2012b; Hong, 2014). De là à réduire la politique scientifique arctique de la Chine à un simple instrument politique, il n'y a qu'un pas que certains analystes évoquent (Teeple, 2010 ; Rainwater, 2012; Brady, 2013; Hong, 2014), soulignant que les résultats de recherche semblent maigres par rapport aux sommes consacrées, ou encore que l'essentiel des intérêts pétroliers et gaziers de la Chine se situe dans le secteur sibérien de l'Arctique. Ce parallèle pourrait être tentant, mais il faut se garder d'interpréter hâtivement les programmes scientifiques arctiques chinois. D'une part, le Polar Research Institute avait, en 2013, nourri activement le projet d'une campagne dans l'Arctique canadien (Lasserre, Huang et Alexeeva, 2013), projet abandonné par 
la suite. D'autre part, si les projets de coopération pétroliers et gaziers se situent tous dans l'Arctique russe, sauf un projet au large de l'Islande, les projets miniers chinois, en revanche, se trouvent dans l'Arctique canadien et groenlandais...

\section{UNE POSTURE DIPLOMATIQUE AGRESSIVE EN ARCTIQUE?}

\section{La fin du mutisme quant à la position officielle de la Chine}

Malgré l'intérêt croissant de la Chine pour l'Arctique, en particulier dans le domaine scientifique mais aussi, de plus en plus, sur les plans diplomatique et économique, aucune stratégie officielle guidant les actions et les déclarations du gouvernement chinois à propos de cette région et de son potentiel (énergétique, maritime, économique, scientifique, militaire, etc.) n'a été publiée jusqu’à maintenant. Pékin dément fermement l'existence d'une telle stratégie et souligne le caractère avant tout scientifique de son intérêt pour l'Arctique (Spears, 2011). Le vice-ministre des affaires étrangères, Hu Zhengyue, a ainsi souligné que la « Chine n'a[vait] pas de politique arctique " au cours d'une conférence tenue au Svalbard en novembre 2009 (cité par Jakobson 2010), même s'il est clair que la Chine nourrit un certain intérêt pour l'Arctique.

Les déclarations des représentants officiels sont très prudentes et traitent principalement des questions de changement climatique et environnementales (Zhang et Ren, 2012). Les changements dans la circulation atmosphérique en provenance de l'Arctique semblent être la cause principale des changements météorologiques importants observés en Chine depuis ces dernières années, notamment la baisse des précipitations en Chine du nord. Ainsi, la région de l'Arctique serait directement liée à la sécurité du développement socio-économique de la Chine, d'où découle l'intérêt du gouvernement chinois envers une meilleure compréhension des mécanismes climatiques de cette région (Qin et Chen, 2011 ; Zhang et Ren, 2012).

Quant aux questions de souveraineté en Arctique et à l'exploitation de ressources naturelles de la région, les déclarations de Pékin sont rares et restent assez vagues. Le gouvernement chinois a longtemps laissé planer une certaine incertitude quant à son intérêt pour ces ressources arctiques : «Puisqu'il n'existe pas d'information fiable sur les réserves de pétrole et de gaz en Arctique, la Chine s'intéresse seulement aux changements climatiques en cette région. Avant de formuler une politique quelconque à ce sujet, il faut d'abord recueillir des informations sur le potentiel minéral et pétrolier [de l'Arctique] » déclarait Xu Shijie, directeur de la division de politique de la Chinese Arctic and Antarctic Administration en 2012 (Xu, 2012).

Le gouvernement chinois ne reconnaissait ni ne niait les droits souverains revendiqués par les États arctiques fondés sur la Convention des Nations unies sur le Droit de la Mer (CNUDM, 1982, entrée en vigueur en 1994), laquelle institue les zones économiques exclusives (ZEE), dans lesquelles les États côtiers détiennent des droits souverains sur les richesses de la colonne d'eau, des fonds marins et du sous-sol, sur 200 miles marins $(370 \mathrm{~km})$ à partir des côtes. Des droits souverains s'établissent aussi pour les ressources du sous-sol sur le plateau continental étendu, au-delà de la limite des 200 miles marins, mais seulement s'il est une extension géologique naturelle du plateau continental physique. Les États arctiques revendiquent tous l'extension de leur zone de juridiction (Steinberg et al., 2010; Bartenstein, 2010), d'où les litiges potentiels entre Russie, Danemark, Canada et États-Unis lorsque les revendications de ces trois derniers seront connues ${ }^{7}$. Là encore, la Chine se retranchait derrière un attentisme prudent dont la formulation entretenait de nombreuses spéculations quant à ses intentions réelles : «La Chine prend note des zones économiques exclusives et des plateaux continentaux étendus des pays riverains de la région arctique, en particulier parce que ces plateaux continentaux doivent encore être définis. La Chine considère [...] le caractère indéterminé des positions juridiques des espaces maritimes de la région arctique... »- précisait Hu Zhengyue, ministre adjoint des Affaires étrangères en 2009 au Svalbard (Jakobson, 2010).

Ces disputes ont été analysées par nombre d'auteurs chinois qui concluent que la communauté internationale doit respecter les recommandations de la CNUDM, bien que, soulignent quelques-uns, certaines zones revendiquées par les pays avoisinant

\footnotetext{
7. La Commission pour les Limites du Plateau Continental est l'organisme des Nations Unies chargé d'évaluer les revendications quant à leur validité géologique, mais n'a pas pour mandat de trancher les litiges frontaliers.
} 
l'Arctique semblent plutôt relever de la juridiction internationale et devraient demeurer ainsi ouvertes à tous (Zhao 2009; Liu et al, 2010) - bref relever de ce que la CNUDM appelle la «Zone». En particulier, le contre-amiral Yin Zhuo est souvent cité depuis qu'il a affirmé que "l'Arctique appartient à tous les peuples du monde et aucun État n'y a de souveraineté » (cité par Chang 2010), sans pourtant que l'on sache trop à quels espaces maritimes l'amiral pensait, ni si ses propos radicaux, certes relayés par l'agence China News Service, sont endossés par le gouvernement.

Toutefois, cette position, si elle devenait la politique officielle de la Chine, serait surprenante, car elle pourrait nuire aux intérêts chinois dans les mers de Chine du Sud et de l'Est. Il serait difficile pour Pékin, qui depuis des années cherche à faire reconnaitre ses revendications maritimes, de justifier l'extension des espaces maritimes chinois mais de nier ce droit aux États arctiques. De même, plusieurs analystes canadiens redoutent que la Chine ne conteste la souveraineté revendiquée par le Canada sur le passage du Nord-Ouest; or, si Pékin conteste le statut d'eaux intérieures affirmé par Ottawa sur ce passage, il sera difficile pour la Chine de défendre une revendication très semblable sur le détroit de Qiongzhou (Lalonde et Lasserre, 2013; Alexeeva et Lasserre, 2015). De fait, en mars 2013, lors d'une rencontre entre chercheurs canadiens (dont F. Lasserre), représentants de l'ambassade du Canada, chercheurs chinois et responsables du Polar Research Institute of China (PRIC), les responsables scientifiques officiels chinois ont souligné que la Chine avait l'intention, à moyen terme, de demander la permission de transit par le Passage du Nord-Ouest pour son brise-glace de recherche, reconnaissant ainsi implicitement la position canadienne. De plus, le gouvernement chinois s'est soumis aux procédures réglementaires canadiennes lors du voyage du brise-glace Xuelong à Tuktoyaktuk (Canada) en 1999 (Pelletier et Lasserre, 2015), et aux procédures russes lors du transit du passage du Nord-Est en 2012 (Gayazova, 2013).

Ce n'est qu'en mai 2013, à la suite de l'admission de la Chine comme observateur permanent au Conseil de l'Arctique, que Pékin a levé toute ambiguïté, lorsque Hong Lei, porte-parole du ministère des Affaires étrangères chinois, a affirmé que « la Chine reconnait la souveraineté, les droits sou- verains et la juridiction des pays arctiques dans la région arctique » (Ministry of Foreign Affairs, 2013). Cette reconnaissance était cependant rendue obligatoire pour devenir observateur permanent depuis l'adoption des critères de Nuuk en 2011 par les membres permanents du Conseil de l'Arctique, parmi lesquels figurent la reconnaissance « de la souveraineté, des droits souverains et de la juridiction » des États arctiques (Déclaration de Nuuk, 2011 ; SAO Report, 2011).

\section{Une diplomatie chinoise active vers l'Arctique européen}

En parallèle aux activités scientifiques chinoises, le gouvernement chinois a aussi développé de nombreux partenariats politiques et économiques avec les pays arctiques, notamment avec le Danemark, l'Islande, la Suède et la Finlande (Pascal 2010). Ainsi, en Islande, depuis la crise financière qui a frappé l'île en 2008, profitant des préoccupations financières d'un gouvernement aux abois ${ }^{8}$, la Chine occupe une place importante dans la vie économique du pays ${ }^{9}$. L'aide financière de Pékin est jugée inestimable par le président actuel de l'Islande, Ólafur Ragnar Grímsson, qui a visité la Chine cinq fois depuis 2007 et qui y fait la promotion de l'Islande en tant que centre logistique potentiel en Arctique (Ward et Hook, 2011).

Lors de la visite officielle du premier ministre chinois, Wen Jiabao, à Reykjavik, en avril 2012, la Chine a signé six accords de coopération avec l'Islande dans les domaines de l'énergie et des sciences et technologies (Nouvel Observateur, 2012; China Daily, 2012), confirmant ainsi le partenariat ébauché dès 2010. En avril 2013, l'Islande et la Chine ont paraphé un accord de libre-échange. En même temps, l'Islande a confirmé son appui à la candidature de la Chine en tant qu'observateur

\footnotetext{
8. La Russie avait aussi tenté de tirer parti des tourments financiers islandais, lorsque Moscou a envisagé de débloquer un prêt de 4 milliards $€$ en octobre 2008, prêt réduit par la suite à 500 millions \$ puis finalement refusé par Moscou en octobre 2009 lorsqu'il devint clair que l'Islande avait obtenu un accord séparé avec le FMI et les pays scandinaves. En janvier 2012, la Chine a promis de soutenir la stabilité financière de l'Islande et sa croissance économique. China's Government Official Portal (2012, 17 janvier), en ligne, [http://english.gov.cn/2012-01/17/content_2046830. $h \mathrm{tm}$ ], c. le 22 octobre 2013.

9. En 2011 , le commerce entre l'Islande et la Chine a atteint le chiffre record de 151 millions de dollars. La Chine exporte en Islande des vêtements, chaussures et produits textiles, et l'Islande approvisionne le marché chinois en poisson, cf. Shanley, 2012.
} 
permanent au sein du Conseil de l'Arctique (Hu, 2012), un appui qui a contribué à l'accession de la Chine, en mai 2013, à ce statut d'observateur. Les analystes répètent à l'envi que la Chine possède la plus grande ambassade à Reykjavik (Wade, 2008; Jakobson, 2010; Beck, 2014), ce qui est exact en termes de taille du bâtiment, mais certainement pas pour le nombre d'employés : au 31 décembre 2014, l'ambassade chinoise comptait 7 employés chinois, tout comme celle du Mexique, d'Allemagne et de France; mais l'ambassade de l'Inde en comptait 8, tout comme celle du Japon; celle de Singapour, 11 ; celle de la Russie, 13, et celle des États-Unis, 14 (ministère des Affaires étrangères d'Islande, 2014) : on ne peut donc affirmer que la délégation chinoise domine le paysage diplomatique islandais.

Le gouvernement chinois a aussi développé de nombreux partenariats politiques et économiques avec les pays arctiques, notamment avec la Norvège (2001), le Danemark (2010). Ainsi, en mai 2010, le Danemark a accueilli la première délégation de commerçants et d'investisseurs chinois qui ont signé des contrats et des lettres d'intention dans les domaines de l'énergie, de l'économie verte, de l'agriculture et de la sécurité alimentaire dont la valeur totale est estimée à plus de 740 millions d'US\$ (Ministry of Foreign Affairs, 2011b).

Les accords signés portent principalement sur le développement de la coopération dans le domaine des recherches sur la navigation en Arctique, de l'exploitation des ressources naturelles et des recherches scientifiques conjointes, mais aussi sur l'appui de la candidature de la Chine auprès du Conseil de l'Arctique. En effet, la Chine était candidate depuis 2008 comme observateur permanent au Conseil, un poste qui ne lui donnerait aucun levier décisionnel, mais lui permettrait de participer aux activités de ce forum intergouvernemental régional qui promeut la coopération et la concertation entre les pays arctiques ${ }^{10}$ (Koivurova, 2009). Après avoir échoué à obtenir ce statut en 2009, la Chine a renouvelé sa demande et a été admise en mai 2013. Le 15 mai 2013, le ministère des Affaires étrangères chinois s'est empressé d'affirmer alors qu'il

10. Le Conseil de l'Arctique réunit depuis 1996 les huit États de l'Arctique, soit le Canada, le Danemark, la Finlande, l'Islande, la Norvège, la Russie, la Suède et les États-Unis. Cette organisation coordonne des discussions sur les aspects environnementaux, économiques et sociaux du développement dans l'Arctique et demeure la principale instance de gouvernance de l'Arctique, même si elle n'a pas de pouvoir décisionnel. reconnaissait la souveraineté des États riverains en Arctique, levant du même coup nombre de soupçons quant aux intentions chinoises à long terme.

La question de la participation de la Chine comme observateur permanent semble un enjeu majeur pour la diplomatie chinoise dans l'Arctique, non pas pour y infléchir la gouvernance de la région - le Conseil de l'Arctique prend très peu de décisions contraignantes pour les membres, et les observateurs n'y ont pas droit de vote - mais bien simplement pour participer et faire entendre la voix de Pékin dans les discussions de coulisses au sujet de l'exploitation des ressources, du régime de navigation et de l'application de la Convention sur le droit de la mer.

\section{DES INTÉRÊTS D'ORDRE ÉCONOMiQue POUR la ChINe?}

\section{Un intérêt pour l'extraction minière qui prend forme}

La Chine ne s'intéresse pas qu'au Conseil de l'Arctique : au Danemark, Pékin a souligné le potentiel minier conséquent du Groenland. Des capitaux chinois importants provenant de Xinye Mining ont été investis dans l'entreprise britannique London Mining, qui devait entamer l'exploitation d'une très importante mine de fer à Isua en 2015 (London Mining, 2011 ; Nunatsiaq News, 2013); l'entreprise a fait faillite en octobre 2014 et il n'est pas certain qu'elle pourra aller de l'avant avec le projet groenlandais.

Au Canada, l'entreprise chinoise Wisco (Wuhan Iron and Steel Co.) envisage l'exploitation d'un gisement de fer majeur au lac Otelnuk (Nunavik) (Les Affaires, 2012). En janvier 2010, l'entreprise minière Jilin Jien Nickel, l'une des plus importantes productrices chinoises de nickel, a fait l'acquisition de Canadian Royalties Inc. et a investi près de 800 millions de dollars en 2012 afin d'exploiter un gisement de nickel situé près de la communauté inuite de Kangiqsujuaq, toujours au Nunavik (Investissement Québec, 2011). MMG envisage d'ouvrir deux mines majeures de zinc et de cuivre près du golfe du Couronnement, dans le Nunavut continental (Izok Lake et High Lake) (Nunatsiaq News, 2012), mais on prévoyait, en novembre 2013, un délai d'un an avant le début des travaux; Jinduicheng 
Molybdenum Group a fait l'acquisition en 2008 de l'entreprise canadienne Yukon Zinc et depuis 2009, Jiangxi Zhongrun Mining et Jiangxi Union Mining explorent des gisements de cuivre et d'or dans le sud groenlandais suite à l'acquisition de l'entreprise britannique Nordic Mining (Lasserre et Têtu, 2014).

Dans la plupart des autres cas, les intérêts miniers chinois se limitent à une participation dans le capital-actions de sociétés, canadiennes principalement, qui développent des projets souvent liés au minerai de fer : outre le projet au lac Otelnuk de Wuhan Iron \& Steel qui possède $60 \%$ des parts de la canadienne Adriana Resources, Wisco détient $20 \%$ de l'américaine Cliffs Natural Resources qui exploite une mine à Fermont dans le Nord du Québec; Wisco et China Minmetals détiennent par ailleurs $25 \%$ et $5 \%$ de l'entreprise canadienne Century Iron Mines, qui développe trois projets dans le nord du Québec. Hebei Iron \& Steel détient $20 \%$ de la canadienne Alderon Iron Ore et s'est engagée à investir 400 millions \$ du projet de mine de fer de Kami estimé à 1,3 milliard; Yunnan Chihong Zinc \& Germanium, qui détient $50 \%$ d'un projet en partenariat avec l'entreprise canadienne Selwyn Zinc, a investi 100 millions \$ dans le projet de Howard Pass Yukon (plomb-zinc).

Nombre de ces projets ont été remis en cause du fait de la chute des cours du minerai de fer au cours de l'automne 2014 : après la faillite de London Mining, cette évolution souligne que, pour les entreprises chinoises comme pour les autres, l'Arctique demeure une région très coûteuse où exploiter un gisement minier. Cependant, il importe de souligner que pour ces multiples projets, les entreprises chinoises ont toujours recherché un partenaire industriel et font avancer leurs intérêts selon les règles du marché.

\section{L'exploitation des hydrocarbures, un rêve coûteux?}

Au cœur de la couverture largement médiatisée de l'exploration des ressources minières et énergétiques de l'Arctique, se trouve la question de l'ampleur des gisements pétroliers et gaziers. Les médias ont largement répercuté l'idée que la région contiendrait des gisements colossaux. Le rapport de 2000 de l'US Geological Service (USGS, 2000) a souvent été mal cité pour lui faire dire que l'Arctique contiendrait environ $25 \%$ des réserves de pétrole encore à découvrir, alors que l'étude de 2000 ne portait pas uniquement sur l'Arctique, mais incluait également des zones boréales. Une étude plus spécifique et rigoureuse, publiée par l'USGS en mai 2008, a estimé les réserves possibles d'hydrocarbures de l'Arctique (soit au nord du cercle arctique) à environ 90 milliards de barils de pétrole, 47261 milliards $\mathrm{m}^{3}$ de gaz naturel, et 44 milliards de barils de condensats de gaz, soit $29 \%$ des gisements à découvrir de gaz, et $10 \%$ des gisements de pétrole (USGS, 2008; Gautier et al., 2009). Une baisse significative depuis le premier rapport de 2000! Même ces chiffres révisés de l'USGS ne font pas l'unanimité : Paul Nadeau, de l'entreprise norvégienne StatoilHydro, a affirmé que les estimations de l'USGS sont de 2 à 4 fois trop optimistes. «Nous croyons que leurs chiffres sont trop élevés. Cela n'importe guère pour les compagnies pétrolières, mais pourrait induire en erreur les gouvernements ${ }^{11}$. » Une étude publiée en 2012 fait état de réserves, pour l'Arctique et l'ex-URSS, d'environ 66 milliards de barils de pétrole, dont $43 \%$ (soit 28,4 milliards de barils) dans l'Arctique; et d'environ 60100 milliards $\mathrm{m}^{3}$ de gaz naturel, dont au moins $58 \%$ se trouveraient dans l'Arctique (soit 34860 milliards $\mathrm{m}^{3}$ de gaz naturel) (USGS, 2012). Avec le temps et l'accumulation de données plus précises, les estimations sur l'ampleur des gisements arctiques s'amenuisent.

Des gisements ont été découverts (nord de l'Alaska, mers de Barents et de Kara) et se pose la question de l'acheminement des volumes produits vers les marchés de consommation. La dimension arctique est aussi au cœur des relations sino-russes, abordée dans le cadre de discussions plus générales sur le partenariat stratégique et énergétique entre les deux pays. Il apparaît que, malgré la méfiance qui peut émailler les relations bilatérales, la Russie entend tirer parti de l'intérêt économique de la Chine pour l'Arctique ${ }^{12}$. Moscou, qui contrôle le passage du Nord-Est et souhaiterait accélérer l'exploitation des ressources naturelles de sa zone arctique, voit en la Chine un client potentiel pour

\footnotetext{
11. «Experts dispute figures on Arctic hydrocarbons », Barents Observer, 13 août 2008, [www.barentsobserver.com], c. le 22 mai 2009.

12. «Китай становится главным покупателем российских нефти и газа » Kitai stanovitsya glavnim pokupatelem rossiskoi nefti i gaza, [La Chine devient l'acheteur principal du pétrole et du gaz russe], News ru.com, 23 octobre 2013, en ligne [www.newsru.com/finance/23oct2013/rosgazkitay.html], c. le 26 octobre 2013.
} 
la Route maritime du Nord (RMN) (Popov, 2010) et un pourvoyeur possible des capitaux nécessaires pour mettre en place ce projet. Toutefois, l'exploitation de ces ressources en milieu arctique nécessite une expertise technologique très avancée ainsi que de l'équipement spécifique (plateformes de forage adaptées) dont la Chine ne dispose pas et que la Russie maîtrise mal (Savelieva et Shiyan, 2010), en témoigne les retards fréquents et les dépassements de coûts dans la mise en exploitation en décembre 2013 du gisement pétrolier de Prirazlomnoye en mer de Petchora, ou du gisement de gaz de Shtokman en mer de Barents, reporté sine die en août 2012 et pour lequel la baisse actuelle des cours des hydrocarbures n'augure pas de réactualisation avant longtemps... Pour Pékin, y accéder suppose des investissements en recherche, développement et maîtrise des savoir-faire de l'ordre de plusieurs milliards de dollars et sur plusieurs années ${ }^{13}$. La Chine et la Russie conduisent également un programme de recherches scientifiques conjoint, lesquelles portent entre autres sur les problèmes techniques et technologiques de la construction de gazoducs et oléoducs dans les conditions arctiques et subarctiques (Du et al., 2010).

Ces difficultés techniques dans l'exploitation des hydrocarbures et les coûts élevés des activités en Arctique ont poussé la Russie à chercher des partenaires à l'étranger, notamment en Chine, afin de faciliter l'exploitation, pour les gisements terrestres pour le moment. Les récentes sanctions occidentales consécutives au conflit en Ukraine, à l'été 2014, renforcent cette ouverture de Moscou envers la Chine, mais aussi l'Inde et le Vietnam notamment. Le partenariat stratégique sino-russe a récemment été réaffirmé alors que la Russie a besoin de partenaires pour financer la coûteuse exploitation des ressources arctiques. Trois compagnies chinoises ont proposé de fournir les capitaux ainsi que la main-d'œuvre nécessaires, China National Petroleum Corporation (CNPC), China National Offshore Oil Corporation (CNOOC) et China Petroleum \& Chemical Corporation Ltd. Dès 2009, un accord avait été paraphé entre CNPC d'une part, Transnweft et Rosneft d'autre part, l'entreprise chinoise accordant des prêts à long terme de 25 mil-

13. Jean-Thomas Bernard, spécialiste en économie de l'énergie, Département de Sciences économiques, université d'Ottawa, correspondance avec les auteurs, 25 octobre 2012 . liards de \$ pour la construction de l'oléoduc Eastern Siberia-Pacific Ocean (ESPO). Un nouvel accord majeur a été signé en juin 2013, CNPC acquérant $20 \%$ des projets de gaz auprès de Novatek, puis un mémorandum en octobre 2013 entre Sinopec et Sibneft, prévoyant la livraison annuelle de 100 millions de tonnes de pétrole russe en Chine. Cet accord, d'une durée de 10 ans, ferait de la Chine le premier acheteur du pétrole russe dans le monde. La plus grande partie du pétrole et du gaz que la Russie pense extraire des gisements arctiques est destinée au marché asiatique, et à la Chine en particulier ${ }^{14}$. En novembre 2014, la Russie a accordé une licence d'exploration gazière à la compagnie chinoise CNOOC (Barents Observer, 2014), qui a déjà conclu un accord semblable avec l'Islande en mars 2014 (IBT, 2014). Enfin, en octobre 2014, un accord majeur a été conclu entre Gazprom et CNPC pour la livraison de gaz naturel (Gazprom, 2014).

Face aux difficultés d'acquisition de la technologie de forage dans l'Arctique, tant pour la Russie et a fortiori que pour la Chine; aux coûts majeurs de la mise en valeur des ressources arctiques; et à l'embargo technique décrété par les Occidentaux suite à la guerre en Ukraine depuis mars 2014, la Chine sera sans doute davantage intéressée à acheter le pétrole extrait dans le cadre de contrats d'achat ou de sociétés mixtes, plutôt que de tenter d'acheter elle-même des sites d'exploitation : la Russie a trop besoin du partenaire chinois pour se passer de son assistance, au risque de développer une

14. Ainsi, la Russie prévoyait de vendre aux Chinois le gaz extrait des nombreux gisements arctiques de la péninsule de Yamal. Cependant, à la suite de la découverte des ressources importantes de gaz de schiste en Chine, Pékin n’a pas accepté de signer immédiatement le contrat avec la Russie, exigeant une baisse des prix du gaz demandés par Moscou, ce que la Russie, confrontée aux efforts de l'Union européenne de diminuer ses achats, à la chute des cours mondiaux, à la chute du rouble (automne 2014) et à l'embargo technique suite au conflit ukrainien, semble de moins en moins en position de refuser. Dans son discours lors de la mise en service du gisement de Bovanenkovo, à Yamal, en octobre 2012, Vladimir Poutine avait souligné que la Russie devait suivre les tendances sur le marché international d'hydrocarbures et développer sa stratégie dans ce domaine en fonction des perspectives à long terme, c'est-à-dire de vendre son gaz de manière plus active à l'est - en Chine et au Japon, cf. Drobinina Ekaterina, « Для кого "Газпром" добывает газ на Ямале? » - Dlia kogo "Gazprom” dobivaet gaz na Yamale [Qui profitera du gaz que Gazprom explore à Yamal ?], BBC Russia, 24 octobre 2012, en ligne : [www.bbc.co.uk/russian/business/2012/10/121024_bovanenkovo_gazprom_launch.shtml]. c. le 30 octobre 2012; Skabeeva, Olga, « Лидеры в Арктике: новое месторождение обеспечит весь экспорт газа в Европу » - Lideri v Arktike: novoe mestorogdenie obespetchit ves eksport gaza v Evropu [Les leaders en Arctique : le nouveau site assurera la totalité d'exportations du gaz en Europe] , Vesti.ru, 23 octobre 2012, en ligne: [www.vesti.ru/doc.html?id=940270\&cid=6], c. le 30 octobre 2012. 
réelle dépendance envers le marché et les capitaux chinois. Les entreprises chinoises ne se limitent pas à la coopération avec la Russie : CNOOC a signé un accord de coopération avec les sociétés islandaises Petoro et Eykon Energy en novembre 2013 pour l'exploration du secteur Dreki du plateau continental islandais. À l'instar des activités minières, on constate que les sociétés pétrolières chinoises traduisent leur intérêt par la signature d'accords de partenariat dans les cadres juridiques et de marché des zones qu'elles ciblent : il n'y a pas de tentative d'intimidation, contrairement aux échos de certains médias.

Par ailleurs, à partir de 2011 , la découverte d'importants gisements de gaz et de pétrole de schiste en Chine a considérablement accru les réserves locales en hydrocarbures, mais ces gisements sont eux aussi coûteux à exploiter et posent de sérieuses questions environnementales, notamment de par les grands volumes d'eau nécessaires pour assurer leur exploitation, dans un milieu très aride : ces découvertes remettront-elles en cause l'intérêt de la Chine pour les hydrocarbures arctiques?

\section{La navigation commerciale dans l'Arctique}

Dans la littérature académique chinoise, comme dans les réflexions occidentales sur les raisons de l'intérêt chinois pour l'Arctique, la navigation tient une place importante. Qu'il s'agisse des Occidentaux ou des Chinois, la potentielle ouverture de routes maritimes plus courtes entre Asie et Atlantique serait une source suffisante d'intérêt marqué pour la Chine. Ainsi, Yang Huigen, directeur général du Polar Research Institute de Shanghai, a-t-il estimé que 5 à $15 \%$ du commerce international de la Chine transiterait par la Route maritime du Nord (RMN, nom commercial du segment du passage du Nord-Est entre les détroits de Kara et de Béring), au nord de la Sibérie, à l'horizon 2020 (The Economist, 2014) (figure 1).

Certes, des expériences sont tentées, essentiellement avec le transport des matières premières exploitées dans la région arctique. Ainsi, la première tentative de transporter les hydrocarbures russes vers la Chine en utilisant la RMN a été entreprise en août 2010. Le tanker Baltica, accompagné d'un brise-glace russe, a mis 27 jours pour livrer les 70000 tonnes de condensat de gaz naturel de Mourmansk à Ningbo, au nord-est de la province chinoise de Zhejiang. En supposant une vitesse constante de 14 nœuds, il aurait mis environ 38 jours par Suez et Malacca.

Ce premier essai a été suivi par la signature d'un accord sur la coopération à long terme dans le domaine de la navigation arctique et pour le développement de la RMN entre la société russe de transport maritime Sovcomflot et China National Petroleum Corporation (CNPC), en novembre 2010. Cet accord, déclaré officiellement partie intégrante de la stratégie de coopération énergétique sinorusse, a été signé en présence de Igor Setchine, vice-premier ministre de la Fédération de Russie et accessoirement président du conseil d'administration de la compagnie Rosneft, le deuxième producteur de pétrole russe, et de Wang Qishan, vice-Premier ministre du Conseil des Affaires d'État de la RPC. Cet accord souligne que la Chine ne conteste pas la souveraineté revendiquée par Moscou sur les eaux intérieures des archipels arctiques russes. Il lui serait par conséquent difficile de contester par la suite la position canadienne, très semblable à la position russe.

En plus des conventions déjà existantes, cet accord détermine les modalités de l'utilisation conjointe du potentiel passage du Nord-Est, qu'il s'agisse de transit ou de transport des hydrocarbures en provenance des gisements pétrogaziers arctiques, soulignant l'intérêt mutuel de cette route : Moscou y voit le développement potentiel d'un lucratif partenariat, et Pékin une route rapide pour acheminer les matières premières dont la Chine a besoin. Moscou s'efforce de promouvoir la RMN comme route maritime internationale depuis 1991. En 2011 et 2012, plusieurs vraquiers ont transporté du minerai de fer chargé à Mourmansk ou à Kirkenes (Norvège) vers des ports chinois, en transitant par la RMN, et plusieurs pétroliers et méthaniers ont fait de même entre Vitino (mer Blanche) et la Chine (Northern Sea Route Administration, 2012).

Ainsi, les efforts russes pour développer le trafic maritime international le long de la RMN commencent-ils à porter leurs fruits. De 4 transits en 2010, on est passé à 34 en 2011, puis 46 en 2012, 71 en 2013, mais 31 en 2014 (NSRA, 2011-2014) : des chiffres loin du trafic de Panama (11947 transits en 2014), Suez (17 148 transits en 2014) ou 


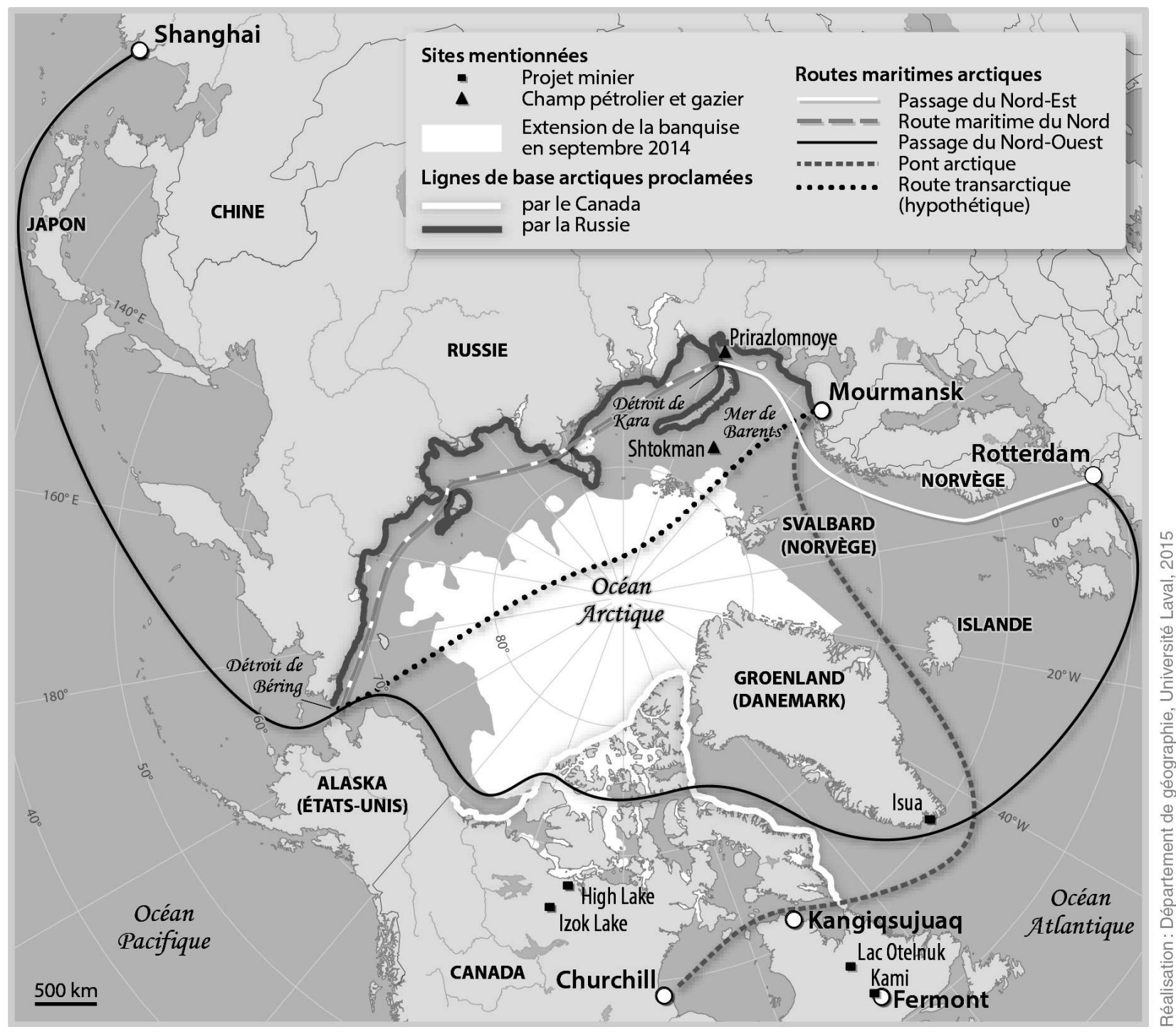

Figure 1 : Routes maritimes potentielles en Arctique Potential maritime routes in the Arctic

de Malacca (77973 transits de navires de plus de 300 t en 2014), certes en croissance sauf pour la brutale chute de 2014, et essentiellement alimentés par l'exportation des ressources naturelles arctiques vers les marchés ultimes, Europe et Asie : il y a peu de transit pur dans ces statistiques russes (Moe 2014; Humpert 2014). Cependant, les entreprises de navigation commerciale chinoises ne se bousculent guère dans l'Arctique : tout le trafic est entre les mains de sociétés russes ou européennes, ce qui confirme le faible intérêt pour la navigation arctique des armateurs chinois (Lee, 2012). Lors d'une série d'entrevues menées par les auteurs de l'automne 2013 à l'été 2014 auprès de 31 transporteurs maritimes chinois majeurs ${ }^{15}$, seuls

15. Cinq entreprises d'État : COSCO; China Merchants Energy Shipping Co; China Shipping Bulk; China Shipping Tanker et CSCL; trois sociétés mixtes d'État : Chipolbrok; China Shipping Development, China LNG CLSICO; 23 entreprises privées : Pacific Glory; Dandong Shipping Group; Evertop Intel Shipping; GMT Shipping; Guangxi Xin'ao Ocean Shipping; Harmony Maritime Inc; Hong Union Shipping; King Far East Shipping; Lufeng Shipping; Maritime Shipping Co.; Nanjing Henglong Shipping Co; Ningbo Jun Hao Ocean Shipping; Ningbo Silver Star; Shandong Ocean Shipping; Shangdong Mou Ping Ocean Shipping; SITC Shipping; Suns International Shipping Co; Tianjin Harvest Shipping Co.; Tong Li Shipping; Uniwill Shipping Co; West Line Shipping; Westline Shipping Co. Ltd Dry Bulk; Winland Shipping; Zhongchang Marine Shipping Co. Enquête menée par Linyan Huang, candidate au doctorat de géographie (Univ. Laval), sous la direction de F. Lasserre, sept. 2013 - août 2014. 
Cosco, China Shipping Development, China LNG CLSICO et Tong Li affirmaient leur intérêt pour les routes arctiques. Cosco, un géant chinois du transport maritime, reconnaissait le caractère incertain de la rentabilité de celles-ci, tandis que China Shipping Development et China LNG CLSICO s'intéressaient aux projets de gaz naturel de la péninsule de Yamal, donc du trafic de destination lié aux ressources.

De fait, la Chine semble davantage s'intéresser aux routes arctiques en ce qu'elles permettent d'accéder à un bassin supplémentaire de ressources naturelles, ressources qu'elle envisage d'acquérir selon les mécanismes du marché; davantage qu'aux possibilités de transit, lequel semble globalement peu intéresser les transporteurs maritimes, occidentaux comme asiatiques (Lasserre et Pelletier, 2011). De ce point de vue, la stratégie chinoise se montre opportuniste : il s'agit d'explorer les pistes tant en matière d'accès aux ressources qu'à des routes commerciales, sachant que dans les deux domaines, l'Arctique n'est qu'une option parmi d'autres : en matières de ressources, les compagnies chinoises sont beaucoup plus actives en Asie centrale et en Afrique; en matière de transport, la Chine investit bien davantage dans le développement d'un lien ferroviaire vers l'Europe, qui présente de plus l'avantage de contourner la Russie et de produire des retombées politiques certaines en Asie centrale (Huang, Lasserre et Alexeeva, 2014; Hong, 2014 ; Bennett, 2014). Toutefois, la presse chinoise a annoncé en septembre 2012 la conclusion d'un accord entre les autorités russes et Cosco, pour étudier la rentabilité de routes commerciales de transit via la zone arctique russe (Zhong, 2013). Le premier navire de Cosco, non pas un porte-conteneur comme souvent rapporté dans les médias ou la littérature scientifique, mais un navire gros porteur polyvalent, le Yong Sheng, a quitté le port de Dalian, dans la province de Liaoning au Nord-Est de la Chine, le 8 août 2013, pour rallier l'Europe. Signe du début de l'exploitation commerciale du passage du NordEst par les Chinois, ou expérience politique avant tout, Cosco étant une entreprise d'État qui, par ailleurs, n'affichait pas un enthousiasme majeur pour les routes arctiques? Toujours est-il que le transit du Hong Xing, annoncé aussi pour l'été 2013, n'a pas eu lieu (NSRA, 2013); que l'expérience du Yongsheng n'a pas été renouvelée en 2014, de même que le voyage du brise-glace de recherche Xuelong annoncé pour l'été 2014 à travers le passage du Nord-Ouest (Lasserre, Huang et Alexeeva, 2013) n'a pas eu lieu, la campagne arctique de l'été 2014 se concentrant plutôt dans les mers de Béring et des Tchouktches.

\section{Conclusion}

Malgré la présence croissante de la Chine en Arctique et l'ancienneté des programmes de recherche, l'affirmation d'une volonté politique est un fait très récent, dont de nombreux éléments restent encore à découvrir et à étudier, car Pékin n'a pas encore articulé de doctrine officielle en la matière. L'analyse de l'évolution de la politique de Pékin envers l'Arctique durant ces quinze dernières années nous a cependant permis de souligner l'existence d'une stratégie que la Chine est en train de mettre progressivement en place afin de défendre ses intérêts dans cette partie du monde.

D’une part, la Chine a réalisé un vaste programme de recherches polaires et a mis en place une vraie structure de gestion des activités chinoises en Arctique en renforçant ainsi sa présence dans cette région. D'autre part, en développant des relations avec les pays entourant l'océan Arctique et en participant de manière active aux débats internationaux au sujet de l'avenir de l'Arctique et du rôle de cette région dans le développement mondial, la Chine a réussi à se faire reconnaître comme l'un des acteurs principaux sur la scène arctique tout en n'ayant aucun accès géographique à cette région. Bien qu'il reste encore beaucoup d'inconnues dans l'équation Chine-Arctique, il semble que la Chine soit parvenue à atteindre un premier objectif dans ce dossier international : faire entendre sa voix dans la gouvernance régionale et disposer d'options sur le développement des ressources du marché via les mécanismes du marché. Enfin, il est certain que la Chine s'intéresse aux ressources naturelles et au potentiel en matière de transport maritime que présente l'Arctique : une intense activité diplomatique de la Chine et un fort dynamisme de ses entreprises dans la région s'efforcent de matérialiser les intérêts chinois, mais en cela, la Chine ne manifeste pas de comportement ni menaçant, ni différent de nombreux autres acteurs internationaux. 


\section{Bibliographie}

Alexeeva O., Lasserre F., 2015. Quelle stratégie pour la Chine en Arctique?, dans Mottet É., Courmont B., Lasserre F. (dir.). La Chine et le Monde. Quelles nouvelles relations, quels nouveaux paradigmes?, Québec, Presses de l'Université du Québec, 301 p., p. 271-292.

Alexeeva O., Lasserre F., 2012a. China and the Arctic, Arctic Yearbook 2012, University of Akureyri, p. 80-90.

Alexeeva O., Lasserre F., 2012b. Le Dragon des Neiges. Les stratégies de la Chine en Arctique. Perspectives chinoises 3, CEFC, Hongkong, p. 61-68.

Barents Observer, 2014. In Russia-China alliance, an Arctic dimension. 14 novembre, [http://barentsobserver.com/en/arctic/2014/11/russia-china-alliance-arctic-dimension-14-11].

Bartenstein K., 2010. Le fond marin arctique : convoitises et confusions, in LAsserre F. (dir.), Passages et mers arctiques. Géopolitique d'une région en mutation, Québec, PUQ, p. 291-318.

BarentsObserver, 2008. Experts dispute figures on Arctic hydrocarbons. Barents Observer, August 13.

BarentsObserver, 2011. Less Russian oil around the coast of Norway, $1^{\text {er }}$ décembre.

BarentsObserver, 2013. China boosts Arctic research, 7 juin.

Beck Andrea, 2014. China's strategy in the Arctic: a case of lawfare?, The Polar Journal, 4:2, p. 306-318, doi : 10.1080/2154896X.2014.954886.

Brady A.M., 2010. China's Rise in Antarctica?, Asian Survey, 50(4), p. 759-785.

Brady A.M., 2012. Polar Stakes : China's Polar Activities as a Benchmark for Intentions, China Brief, 12(14), 11-15.

Brady A.M., 2013. China's Antarctic interests, in Brady A.M. (dir.), The Emerging Politics of Antarctica, Londres, Routledge, p. 31-49.

Bennetr M., 2014. China's Silk Road plans could challenge Northern Sea Route. Cryopolitics, Arctic News \& Analysis, 29 décembre, [http://cryopolitics.com/2014/12/29/chinassilk-road-plans-could-challenge-northern-sea-route/].

Callus A., Golubrova K., 2012. Rosneft to buy TNK-BP in two-step deal. The Globe and Mail, 22 octobre, en ligne : [www.theglobeandmail.com/report-on-business/internationalbusiness/european-business/rosneft-to-buy-tnk-bp-in-two-stepdeal/article4627976/], c. le 30 oct. 2012.

Campbell C., 2012. China and the Arctic: Objectives and Obstacles, U.S.-China Economic and Security Review Commission Staff Research Report, Washington, DC, 11 p.

Centre de recherches polaires de Chine, 2007. Zhongguo beiji yahjiu zhongxin jianjia [中国极地研究中心简介] Présentation du Centre de Recherches polaires de Chine, Zhongguo beiji yahjiu zhongxin jianjia [中国极地研究中心], [www.china.org.cn/english/features/PolarResearch/168048. htm], c. le 3 novembre 2013.

Chang G., 2010. China's Arctic Play, The Diplomat, 9 mars, [http://the-diplomat.com/2010/03/09/china\%E2\%80\%99sarctic-play/], c. le 25 nov. 2011.

Chen L. et al., 2003. Yingxiang Beiji diqu xunshu bianhuade yixie guanjian guocheng yanjiu [影响北极地区迅速变化
的一些关键过程研究] - Étude des principaux facteurs exerçants une influence sur les changements rapides en Arctique. Jidi yanjiu [极地研究] - Chinese Journal of Polar Research, 15(4), p. 283-302.

Chen Z., 2012. China's South China Sea Policy and its implications for the Canadian Arctic, communication, colloque La Chine et l'Arctique, Montréal, OPSA-UQÀM, 30 avril.

Cheng B., 2011. Arctic Aspirations, Beijing Review, 34, 25 août.

China Daily, 2012. China has a key role in safeguarding the Arctic, 29 juin, p. 9.

China Daily, 2014. New icebreaker planned by 2016: officials, 6 janvier, [www.chinadaily.com.cn/china/2014-01/06/ content_17216579.htm].

Déclaration de Nuuk, 2011. Nuuk Declaration on the occasion of the Seventh Ministerial Meeting of the Arctic Council, Nuuk, Groenland, 12 May 2011 . [http://www.arctic-council.org] (consulté le 17 octobre 2011).

Drobinina E., 2012. Для кого “Газпром” добывает газ на Ямале? - [Qui profitera du gaz que Gazprom explore à Yamal ?]. BBC Russia, [www.bbc.co.uk/russian/business/2012/10/121024_bovanenkovo_gazprom_launch.shtml]. c. le 30 octobre 2012 .

Du M. [杜明俊], Ma G. [马贵阳], Chen X. [陈笑寒], 2010. Bingtuqu maidi reyou guandao tingshu wenjiang shuzhi mosi [冻土区埋地热油管道停输温降数值模拟]-Simulation numérique des chutes de températures dans un oléoduc chaud pendant un arrêt complet dans les régions de pergélisols. Tianranqi yu shiyou [天然气与石油] - Natural Gas and Oil, 28(4), p. 54-57.

Edmonton Journal, 2007. Who will guard our gaping back door?, 18 novembre.

Dutton P., 2012. Law of the Sea for the 21 st century, in DutTON P. et al. (dir.), Twenty-first Century Seapower. Cooperation and conflict at sea, Londres, Routledge, p. 262-280.

Gautier D. L. et al., 2009. Assessment of Undiscovered Oil and Gas in the Arctic, Science, 324, 29 mai, p. 1175-1179.

Gayazova O., 2013. China's Rights in the Marine Arctic, The International Journal of Marine and Coastal Law, 28(1), p. 61-95.

Gazprom, 2014. Gazprom and CNPC sign Technical Agreement on gas supplies via eastern route. Communiqué, 13 octobre, [www.gazprom.com/press/news/2014/october/ article203444/].

Grupta A., 2009. Geopolitical implications of Arctic meltdown, Strategic Analysis, 22(2), p. 174-177.

Holmes S., 2008. Breaking the Ice: Emerging Legal Issues in Arctic Sovereignty, Chicago Journal of International Law, 9(1), p. 323-352.

Hong N., 2014. Emerging interests of non-Arctic countries in the Arctic: a Chinese perspective, The Polar Journal, 4:2, p. 271-286.

Huang L., Lasserre F., Alexeeva O., 2014. Is China's interest for the Arctic driven by Arctic shipping potential?, Asian Geographer, doi : 10.1080/10225706.2014.928785, p. 1-13.

Hu Y., 2012. China, Iceland pledge further cooperation during Wen's visit, China Daily, [www.chinadaily.com.cn/ 
china/2012-04/21/content_15105506.htm], c. le 4 mai 2012.

Humpert M., 2014. Arctic Shipping: An Analysis of the 2013 Northern Sea Route Season, Arctic Yearbook 2014, p. 195205.

IISS, 2014. China's strategic Arctic interests, Strategic Comments, 20:2, i-ii, doi: 10.1080/13567888.2014.914777.

IBT, International Business Times, 2014. China National Offshore Oil Corp (CNOOC) First Chinese Firm Licensed To Explore Arctic Oil And Gas Resources. 7 mars, [www. ibtimes.com/china-national-offshore-oil-corp-cnooc-firstchinese-firm-licensed-explore-arctic-oil-gas-resources].

Investissement Québec, 2011 , Jilin Jien Nickel porte à $800 \mathrm{M} \$$ ses investissements dans le Nord québécois, InvestQuébec Express, 3(7), septembre.

Jakobson L., 2010. China Prepares for an Ice-Free Arctic, SIPRI Insights on Peace and Security, 2, 16 p.

JiA Y., 2010. Beiji diqu lingtu zhuquan he haiyang quanyi zhengduan tanxi 北极地区领土主权和海洋权益争端探 析 [An Analysis of the Dispute over Arctic Region's Territorial Sovereignty and Maritime Rights], 中国海洋大学学 报 (社会科学版), Journal of the Ocean University of China (Social Sciences), 1.

Peng J., Wegge N., 2014. China and the law of the sea: implications for Arctic governance, The Polar Journal, 4(2) p. 287-305.

Keyuan Z., 1993. China's Antarctic policy and the Antarctic Treaty system, Ocean Development E International Law, 24(3), p. 237-255.

Koivurova T., 2009. Limits and possibilities of the Arctic Council in a rapidly changing scene of Arctic governance, Polar Record, 46(2), p. 146-156.

Lalonde S., Lasserre F., 2013. The Position of the United States on the Northwest Passage : Is the Fear of Creating a Precedent Warranted?, Ocean Development and International Law (44)1, p. 28-72.

Lasserre F. (dir.), 2010. Passages et mers arctiques. Géopolitique d'une région en mutation, Québec, Presses de l'Université du Québec, 516 p.

Lasserre F., 2010b. Géopolitiques arctiques : pétrole et routes maritimes au cœur des rivalités régionales?, Critique Internationale, 49, p. 131-156.

Lasserre F., Pelletier S., 2011 . Polar super seaways? Maritime transport in the Arctic: an analysis of shipowners' intentions, Journal of Transport Geography, 19, p. 1465-1473.

Lasserre F., TÊtu P.-L., 2014. La Chine à la conquête de l'Arctique?, Diplomatie - Grands Dossiers 20 (Paris), p. 72-73.

Lasserre F., Huang L., Alexeeva O., 2013. Science et politique arctiques en Chine. Éclairages de la série de séminaires sino-canadiens, Monde chinois, Nouvelle Asie 34, p. 157-159.

Lee S.W., 2012. Potential Arctic Shipping - Change, Benefit, Risk, and Cooperation, Proceedings of the 2012 North Pacific Arctic Conference, Honolulu, 8-10 août.

Les Affaires, 2012. La Chine affirme sa présence dans l'économie minière du Québec, 28 avril, [http://tinyurl.com/LesAffaires-Mines].
Lı Z. [李振福], 2009. Beiji hangxiande zhongguo zhanluë fenxi [北极航线的中国中战略分析] - L'analyse de la stratégie chinoise en matière de route maritime du Nord, Zhongguo ruankexue [中国软科学] - China Soft Science, 1, p. 1-7.

Li Z., 2009b. Obstacles to China s Participation in the International Arctic Route Mechanism and Countermeasures, Navigation of China, (2), p. 98-103.

Li Z. [李振福], 2009c. Dihuan zhengzhi lilun yu Beiji hangxian diyuan zhengzhi lilun zhashe [地缘政治理论演变与北极航 线地缘政治理论假设] - Évolution et l'analyse de la géopolitique des routes maritimes en Arctique, Shijie Dili Yanjiu [世界地理研究] - World Regional Studies, 19(1), p. 6-13.

Liu H., Dong Y., 2010. Zhongguo haiyang quanyi falv baozhang shiye zhong de jidi wenti yanjiu 中国海洋权益法律保障视 野中的极地问题研究 [Polar Research from the Perspective of Legal Protection of China's Maritime Rights and Interests], 中国海洋大学学报 (社会科学版) - Journal of the Ocean University of China (Social Sciences), 5.

Liu H., Dong Y., Hou Y. [刘惠荣, 董跃, 侯一家], 2010. Baozhang woguo Beiji kaocha jixian guanyi falü tujing chushen [保障我国北极考察及相关权益法律途径初探] - L'approche légale pour protéger pour défendre les intérêts et les droits chinois en Arctique, Zhonguo haiyang daxue xuebao [中国海洋大学学报] - Journal of Ocean University of China, 6, p. 1-4.

Liu H., YANG F. [刘惠荣, 杨凡], 2010. Beiji shengtai baohu falü wenti yanjiu [北极生态保护法律问题研究] Recherche sur les enjeux du droit environnemental arctique du point de vue du droit international, Pékin, Beijing Shi, $251 \mathrm{p}$.

Liu X., 2008. Shixi eluosi de beiji zhanlue [试析俄罗斯的北极 战略] [An Analysis of the Arctic Strategy of Russia]. 东北 亚论坛 - Northeast Asia Forum 18(6).

London Mining, 2011 , ISUA Iron Ore Mine Project, Greenland, Londres.

Lu J. [陆俊元], 2010. Dangjin Beiji diyuan zhengzhi geju tezheng fenxi [当今北极地缘政治格局特征分析] - Analyse des caractéristiques des tendances géopolitiques dans l'Arctique à l'heure actuelle, Shijie dili yanjiu [hei] - World Regional Studies, 19(1), p. 1-5.

Lu J., 2011. Zhongguo zai beiji diqu de zhanlue liyi fenxi [中国 在北极地区的战略利益分析 - 非传统安全视角] [Analysis on China's strategic benefit in the Arctic - from non-traditional security perspective], Jiangnan shehui xueyuan xuebao [江南社会学院学院]. South Yangzi River Human Science University Journal, 4.

Mei H., Wang Z., 2010. Beiji haiyu falv diwei zhengduan jiqi jiejue [北极海域法律地位争端及其解决] [The Dispute of the Legal Status of the Arctic Waters and Its Solution], 中 国海洋大学学报 (社会科学版), Journal of the Ocean University of China (Social Sciences), 1.

MREN, 2012, Chinese to build Polar RV, Maritime Reporter $\&$ Engineering News (MREN), 8, p. 55.

Ministère des Affaires Étrangères d'Islande, 2014. Diplomatic and Consular List, December 2014. Foreign Missions to Iceland, [www.mfa.is/diplomatic-missions/foreignmissions/], Reykjavik. 
Ministry of Foreign Affairs of the People's Republic of China, 2010. China's View on Arctic Cooperation, [http:// tinyurl.com/MFA-PRC], c. 8 décembre 2011.

Ministry of Foreign Affairs of the People's Republic of China, 2011. Director General Huang Huikang Meets with Canadian Assistant Deputy Minister of Foreign Affairs Christie, [www.fmprc.gov.cn/eng/wjb/zygy/gyhd/t858972. htm], c. le 8 décembre 2011.

Ministry of Foreign Affairs of the People's Republic of China, 2011 b. China and Denmark Bilateral Relations, sur [www.fmprc.gov.cn/eng/wjb/zzjg/xos/gjlb/3281/], c. le 22 oct. 2013.

Ministry of Foreign Affairs of the People's Republic of China, 2013. Foreign Ministry Spokesperson Hong Lei's Remarks on China Being Accepted as an Observer of the Arctic Council, 15 mai, [www.fmprc.gov.cn/eng/xwfw/s2510/ t1040943.shtml], c. le 21 octobre 2013.

Moe Arild, 2014. The Northern Sea Route: Smooth Sailing Ahead? Strategic Analysis, 38(6). p. 784-802.

New York Times, 2012. Race Is On as Ice Melt Reveals Arctic Treasures, 18 septembre.

News.ru, 2013. Китай становится главным покупателем российских нефти и газа - Kitai stanovitsya glavnim pokupatelem rossiskoi nefti i gaza, [La Chine devient l'acheteur principal du pétrole et du gaz russe], 23 octobre, en ligne, [www.newsru.com/finance/23oct2013/rosgazkitay.html], c. le 26 octobre 2013.

NSRA, Northern Sea Route Administration, 2011, 2012, 2013, 2014. NSR Transit Traffic Statistics, [www.arctic-lio. com/nsr_transits].

Nouvel Observateur, 2012. Arctique, géothermie, droits de l'Homme abordés en Islande avec le Premier Ministre chinois, [http://tempsreel.nouvelobs.com/monde/20120421. AFP3988/arctique-geothermie-droits-de-l-homme-abordesen-islande-avec-le-premier-ministre-chinois.html], c. 29 avril 2012.

Nunatsiaq News, 2012. MMG forges ahead with Izok zinc-copper mine proposal, 4 sept., [http://tinyurl.com/Nunatisiaq], c. le 29 oct. 2012.

Nunatsiaq News, 2013. Huge Isua iron mine under development in western Greenland, 19 septembre, [http://tinyurl. com/isua-iron], c. le 29 oct. 2013.

Pascal C., 2010. Global Warring. How Economical, Economic and Political Crises Will Redraw the World Map, New York, Palgrave McMillan, 280 p.

People's Daily Online, 2011. China's new polar icebreaker to launch in 2013. [http://english.peopledaily.com. cn/90001/90776/90881/7416709.html], c. 24 avril 2012.

Pelletier S., Lasserre F., 2015. Intérêt de la Chine pour l'Arctique : analyse de l'incident entourant le passage du brise-glace Xue Long en 1999 à Tuktoyaktuk, Territoires du Nord-Ouest, Monde chinois, Nouvelle Asie, 41, accepté, à paraitre.

Peresipkin V.I., IAKovLEV A.N., 2006. Северный морской путь в проблеме международных транспортных коридоров [La route maritime du nord dans les réseaux de transport internationaux], Транспорт Российской Федерации -
Transport Rossï̌skoi Federatsii [Transport de la Fédération de la Russie], 3, 30-35.

Popov V.A., 2010. Перспективы развития портов Арктики и Северного морского пути [Les perspectives du développement des ports en Arctique et du celui de passage du NordEst], Развитие порто - Razvitie portov [Développement des ports], 5, 12-15.

Qin Q., Chen Y., 2011 . The Post-Cold War International Cooperation in the Arctic Region, China International Studies, 4, p. 138-155.

Rainwater S., 2012. Race to the North: China's Arctic Strategy and Its Implications', Naval War College Review, 66(2), p. 62-82.

Ren X., Li Y., 2008. Beibingyang zhuquan zhi jue yu Zhongguo guoji zeren xianxi [北冰洋主权之争与中国国际责任 浅析] - Analyse des problèmes de souveraineté en océan Arctique et responsabilité internationale de la Chine dans ce dossier. Lanfang shifang xue yuan xuebao [廊坊师范学院 学报] - Journal of Lanfang Teachers College, 24(4), p. 66-69.

SAO Report, 2011 . Senior Arctic Officials (SAO) Report to Ministers, Nuuk, Greenland, mai, [www.arctic-council.org] (c. le 19 novembre 2014).

Savelieva S.B., Shiyan G.N., 2010. Арктика: укрепление геополитических позиций и экономическое развитие [L'Arctique : le renforcement des positions géopolitiques et le développement économique], Вестник МГУ - Vestnik MGU [Courrier de l'Université d'État de Moscou], 13(1), p. 115-119.

Shanley M., 2012. China's Wen in Iceland, eyes on Arctic riches. Reuters, 20 avril, [www.reuters.com/article/2012/04/20/uschina-europe-idUSBRE83JOM920120420], c. le 22 oct. 2013.

SHi C. [史春林], 2010. Beibingyang hangxian kaitong dui Zhongguo jingji fazhande zuoyong ji Zhongguo liyong duice [北冰洋航线开通对中国经济发展的作用及中国利用对 策] - L'utilisation et le rôle des routes maritimes en Arctique pour le développement économique de la Chine, Jingji wenti tansuo [经济问题探索] - Inquiry Into Economic Issues, 8, p. 47-52.

Skabeeva O., 2012. Лидеры в Арктик, новое месторождение обеспечит весь экспорт газа в Европу [Les leaders en Arctique : le nouveau site assurera la totalité d'exportations du gaz en Europe], Vesti.ru, en ligne: [www.vesti.ru/doc. html?id=940270 \& cid=6], c. le 30 octobre 2012.

Spears J., 2011. The Snow Dragon Moves into the Arctic Ocean Basin, China Brief, 11(2), Jamestown Foundation, c. le 4 mai 2012.

Staalesen A., 2012. Prime Minister Wen Jiabao is ready to tour Sweden and Iceland in a bid for his country's permanent observer status. Barents Observer, 17 avril.

Steinberg P.E., Tasch J., Fabiano S.J., Shields R., 2010. Contested Sovereignty in a Changing Arctic, Annals of the Association of American Geographers, 100(4), p. 9921002.

TeEple Nancy, 2010. L'histoire des intrusions dans l'Arctique canadien, en bref, Le Journal de l'Armée du Canada, 12(3), p. 52-77. 
The Economist, 2014. China Is Making a Huge Arctic Play Even Though It Has No Claim, 13 juillet, [www.businessinsider.com/china-is-making-a-huge-arctic-play-2014-7].

USGS, United States Geological Survey, 2000. World Petroleum Assessment 2000 - Description and Results, [http:// pubs.usgs.gov/dds/dds-060], c. le 20 octobre 2008.

USGS, United States Geological Survey, 2008. CircumArctic Resource Appraisal: Estimates of Undiscovered Oil and Gas North of the Arctic Circle, [http://pubs.usgs.gov/ fs/2008/3049/fs2008-3049.pdf], c. le 25 octobre 2008.

USGS, United States Geological Survey, 2012. An Estimate of Undiscovered Conventional Oil and Gas Resources of the World, 2012. Fact Sheet 2012-3042. [http://pubs.usgs.gov/ fs/2012/3042/fs2012-3042.pdf], c. le 16 décembre 2014.

Wade R., 2008. A Warmer Arctic Needs Shipping Rules. Financial Times, 16 janvier 2008.

WANG X., 1988. Beiji haishui mianjide shikong fenbu jiqi Changjiang shang, zhongyouxiuqi shuliang fenggude guanxi, 北极 海冰面积的时空分布及其与长江上、中游汛期水量丰枯 的关系 [Sur le lien entre la distribution spatiale et temporelle de glace de l'océan Arctique et le débit du cours moyen et bas du Yangzi en période des crues], mémoire de maîtrise en climatologie, Université de Hohai, Chine.

WANG L., 2007. Beibinyang zhuquan zhizheng de qushi [北冰 洋主权之争的趋势] - Trends of Struggling for Sovereignty over the Arctic, 现代国际关系 - Contemporary Internal Relations, 10, p. 17-21.

Ward A., Ноок L., 201 1. Iceland's president welcomes Chinese interest, Financial Times, [http://tinyurl.com/FinancialTimes-Iceland], c. le 21 octobre 2012.

WeEsE B., 2010. Japan latest non-Arctic country to claim stake in North Pole, Toronto Sun, [www.torontosun.com/news/ canada/2010/09/03/15241971.html], c. 11 avril 2012.

WEI J. et al., 2010, Decrease in the $\mathrm{CO}_{2}$ Uptake Capacity in an Ice-Free Arctic Ocean Basin, Science, 329(5991), p. 556-559.

Wright D.C., 2011. The Panda Bear Readies to Meet the Polar Bear : China Debates and Formulates Foreign Policy Towards Arctic Affairs and Canada's Arctic Sovereignty, Canadian Defence \& Foreign Affairs Institute Papers, Calgary, $10 \mathrm{p}$.

Wright D.C., $2011 \mathrm{~b}$. The Dragon Eyes the Top of the World: Arctic Policy Debate and Discussion in China, Naval War
College, Center for Naval Warfare Studies, China Maritime Studies Institute, Newport, RI, 56 p.

Wright D.C., 2013. China's Growing Interest in the Arctic, Journal of Military and Strategic Studies, 15(2), [http://jmss. org/jmss/index.php/jmss/article/view/528].

Wright T.C., 2013. China's New Arctic Strategem: A Strategic Buyer's Approach to the Arctic, Journal of Military and Strategic Studies, 15(1), [http://jmss.org/jmss/index.php/jmss/ article/view/512].

Xu S., 2012. Chinese Arctic and Antarctic AdministrationChina's Activities and Prospecting in the Arctic, colloque La Chine et l'Arctique, Centre d'études des politiques étrangères et de sécurité (CEPES), 30 avril 2012, Montréal, Canada.

YAN Q. [颜其德], 2005. Beiji diqu yu quandqie bianhua [北极地 区与全球变化] - L'Arctique et le changement global, Kexue [科学], Science, 57(3), p.14-17.

Yu T.Y., 2006. Cong haiyang dao haiyang... zai dao haiyang jianada jingying beiji diqu [从海洋到海洋...再到海洋一加 拿大经营北极地区] - From ocean to ocean... and finally to the Ocean - How would Canada run the North, Shijie zhishi 世界知识. - World Knowledge, 23.

ZHANG S. [张胜军], Li X. [李形], 2010. Zhongguo nengyuan anquan yu Zhongguo Beiji zhanluë dingwei [中国能源安 全与中国北极战略定位] - La sécurité énergétique de la Chine et sa position géopolitique en Arctique, Guo ji guancha [国际观察] - International Review, 4, p. 64-71.

Zhang Y., Ren Q., 2012. China defends Arctic research, Xinhua, [http://news.xinhuanet.com/english/china/201201/31/c_122637030.htm], c. le 18 avril 2012.

Zhно Y. [赵渘], 2009. Xianxi Beiji suomian linde falü taozhan jiqi dui woguode yinxiang [浅析北极所面临的法律挑战 及其对我国的影响] - L'analyse des défis légaux en Arctique et son influence sur la Chine, Haiyang kaifa yu guanli [海洋开发与管理] - Ocean Development and Management, 26(3), p. 17-21.

Zhong N., 2013. Arctic trade route opens, China Daily, 10 août, p. 3.

Zhu S., 2011 . Arctic competition heats up as ice melts, Global Times, [www.globaltimes.cn/NEWS/tabid/99/articleTypel ArticleView/articleId /666783/Arctic-competition-heats-upas-ice-melts.aspx], c. le 8 décembre 2011. 